\section{Review Article}

\section{Check for updates}

\section{OPEN ACCESS}

\section{Received: Jan 11, 2018}

Accepted: Feb 1, 2018

\section{Correspondence to} Gianlorenzo Dionigi

Division for Endocrine and Minimally Invasive Surgery, Department of Human Pathology in Adulthood and Childhood "G. Barresi", University Hospital G. Martino, University of Messina, Via C. Valeria Gazzi, Messina 98125, Italy.

E-mail: gdionigi@unime.it

Copyright (C) 2018. Korean Association of Thyroid and Endocrine Surgeons; KATES This is an Open Access article distributed under the terms of the Creative Commons Attribution Non-Commercial License (https:// creativecommons.org/licenses/by-nc/4.0/).

ORCID IDS

Hoon Yub Kim iD

https://orcid.org/0000-0002-6731-3912 Young Jun Chai (iD https://orcid.org/0000-0001-8830-3433 Hui Sun (iD)

https://orcid.org/0000-0002-6492-3459 Gianlorenzo Dionigi (D)

https://orcid.org/0000-0003-0864-6087

\title{
Cost-Effectiveness Estimate for Neural Monitoring in Thyroid Surgery
}

\author{
Hoon Yub Kim (i) ', Young Jun Chai (ii) ${ }^{2}$, Francesco Freni ${ }^{3}$, Özer Makay ${ }^{4}$, \\ Bruno Galletti ${ }^{3}$, Francesco Galletti ${ }^{3}$, Hui Sun (i) $^{5}$, Gianlorenzo Dionigi (i]) 6
}

'KUMC Thyroid Center, Korea University Anam Hospital, Seoul, Korea

${ }^{2}$ Department of Surgery, Seoul Metropolitan Government-Seoul National University Boramae Medical Center, Seoul, Korea

${ }^{3}$ Division for ENT Surgery, Department of Human Pathology in Adulthood and Childhood "G. Barresi", University Hospital G. Martino, University of Messina, Messina, Italy

${ }^{4}$ Division of Endocrine Surgery, Department of General Surgery, Ege University Hospital, Izmir, Turkey ${ }^{5}$ Division of Thyroid Surgery, Jilin Provincial Key Laboratory of Surgical Translational Medicine, China-Japan Union Hospital of Jilin University, Changchun, China

${ }^{6}$ Division for Endocrine and Minimally Invasive Surgery, Department of Human Pathology in Adulthood and Childhood "G. Barresi”, University Hospital G. Martino, University of Messina, Messina, Italy

\section{ABSTRACT}

There is growing evidence for the importance of more detailed cost evaluation of new technologies used in surgery. The innovative impact of technology holds the potential to achieve transformative clinical improvements. Sustainability of innovations is a relatively new concept in health care research and has become an issue of growing interest. Costeffectiveness studies have been the most established and studied methods for evaluation in surgical patients. Limited information exists regarding the cost-effectiveness of new surgical strategies for monitored thyroid surgeries. We describe the recent evidence regarding methods of evaluation of cost-effectiveness structures and function for intraoperative neural monitoring (IONM) in thyroid surgery. Our findings suggest that health economics modeling to inform the design of a cost-effectiveness studies looking at IONM has been demonstrated to be feasible as a method for improving research efficiency.

Keywords: Thyroid surgery; Neural monitoring; Cost-effectiveness analysis

\section{INTRODUCTION}

Intraoperative neural monitoring (IONM) is a device for the management of the recurrent laryngeal nerve (RLN) in thyroid and parathyroid surgery $(1,2)$. The interest in this accessory grown in recent years and more and more monitored procedures are performed (3,4) (Fig. 1). The development of non-invasive IONM device, user-friendly systems, the publication of a prospective randomized trial and defining guidelines for standards, proctoring, research, Societies recommendations, commercial effort contributed to this dissemination (2,5-9). Hospital administrations invest in neural monitoring technology in an attempt to increase volumes and referral of interventions, and perhaps to get some form of medico-legal protection by adopting the IONM. 
Author Contributions

Conceptualization: Hoon Yub Kim, Gianlorenzo Dionigi, Hui Sun; Data curation: Gianlorenzo Dionigi; Formal analysis: Gianlorenzo Dionigi; Investigation: Gianlorenzo Dionigi; Project administration: Özer Makay; Writing - original draft: Hoon Yub Kim, Young Jun Chai, Francesco Freni, Özer Makay, Bruno Galletti, Francesco Galletti, Hui Sun, Gianlorenzo Dionigi.

Conflict of Interest

No potential conflict of interest relevant to this article was reported.

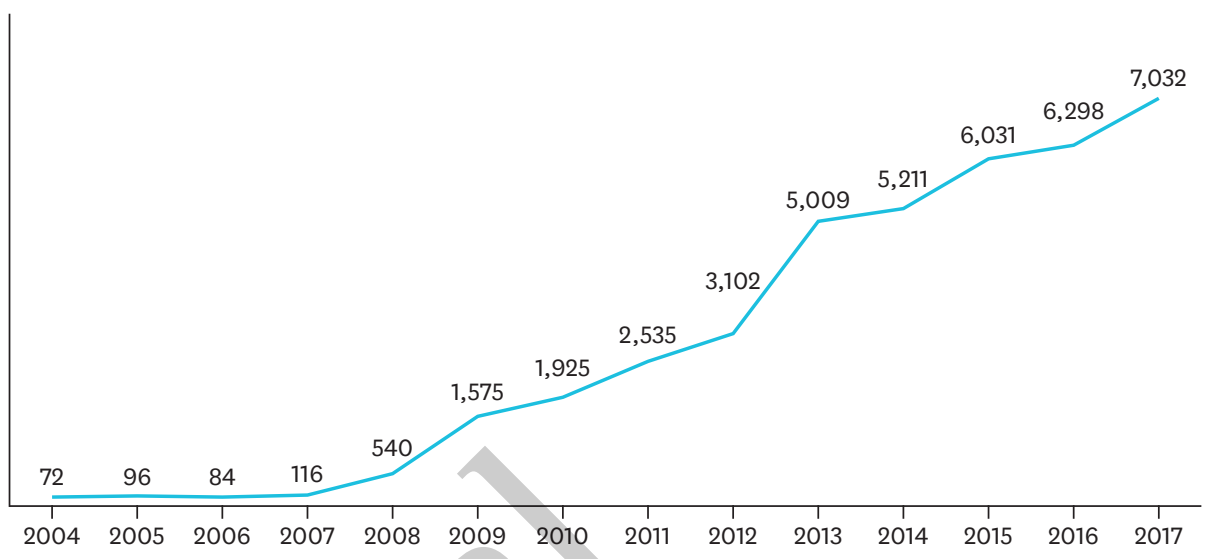

Fig. 1. Prevalence of IONM in Italy. Data from Italian consumption of electromyogram tubes. About 38 to 40,000 thyroidectomies per year are performed in Italy, that is about $16 \%$ are monitored. IONM = intraoperative neural monitoring.

Increase IONM utilization imposes an economic evaluation for a useful information for surgeons, health care professionals, patient, community, and medical device industry. The additional expense of the IONM technique is a concern. There are few data on the economic impact of IONM in thyroid surgery, none for parathyroid interventions (10-16). However, surgeons involved in thyroid procedures, especially in high-volume settings, acquainted the benefits of the technology use.

The aim of this paper is to critically examine current evidence on expense and costeffectiveness studies of IONM.

\section{HOSPITAL AND SURGERY IONM COST}

New technologies in surgery can result an increase in health costs because they are more expensive than previous treatments and because more patients are treated with.

Furthermore, many different formats have been proposed and used nerve monitoring: laryngeal palpation, glottal observation, glottal pressure monitoring, intramuscular electrodes on the vocal cord placed endoscopically, intramuscular electrodes placed through the cricothyroid membrane, surface electrodes positioned on the endotracheal tube (endotracheal tube-based surface electrodes) and needle electrodes (postcricoid surface electrodes) (8). For several reasons, including safety and simplicity, systems that are based on positioned surface electrodes on the endotracheal tube currently represent the most widespread monitoring equipment (8). Further research is necessary to analyze and compare the costs associated with these systems.

\section{Endotracheal tube-based surface electrodes}

Through a micro-costing approach, the thyroidectomy patient-care process (with and without IONM) was analyzed by considering direct costs (staff time, consumables, equipment, drugs, operating room, and general expenses) in an Italian study (16). The data presented can be very specific, unique and of interest mainly for the Italian system and therefore not directly transferable elsewhere, for example in the United States, but definitely interesting for a comparison between the Italian system in thyroid surgery and others. Comparison between costs and the diagnosis related groups (DRGs) fee showed an underfunding of total 
hospitalization costs for all thyroidectomies, regardless of IONM use (16). The main cost drivers are consumables and technologies (25\%), operating room (16\%), and staff $(14 \%)$. Hospitalization costs for a thyroidectomy with IONM range from $€ 3,713$ to $€ 3,770,5 \%-7 \%$ higher than those for traditional thyroidectomy. Estimates on the net cost per procedure of the use of the IONM vary depending on the volume with which the technique is used. These costs are sensitive to the volume of activity, in the sense that as the volume increases, the total hospitalization costs decrease. These differences in total costs are found because costs related to the initial capital investment for IONM technology are spread and amortized over a large number of procedures.

Furthermore, the other technologies used in thyroid surgery have a greater effect on costs than that the IONM. Major economic differences emerge when an energy-based devices is used $(€ 3,969)(16)$. The Authors concluded that the regional DRG tariff for thyroid surgery is barely sufficient to cover conventional surgery costs (16).

Sanguinetti et al. (17) first conducted a retrospective chart review, analysis of surgery time and cost with and without IONM. According to authors, IONM did not reduce the operative time during total thyroidectomies in our experience. Use of IONM increased the cost of each surgery by $€ 450$.

\section{Laryngeal palpation}

Echeverri and Flexon (18) described and analyzed a simple technique with laryngeal palpation for identifying the RLN with a nerve stimulator. A retrospective review of 70 thyroidectomies was performed. The technique was a less costly and time-consuming alternative to intraoperative RLN monitoring (18).

\section{MAGNITUDE vocal cord paralysis (VCP)}

Jeannon et al. (19) in a recent meta-analysis of more than 25,000 patients found post-surgery VCP rate of $9.8 \%$. Francis et al. (20) reviewed post-surgery VCP occurring in over 5,000 Medicare beneficiaries undergoing thyroidectomy and found unilateral VCP rate of $9 \%$.

Bilateral VCP rates are to some extent just as difficult to obtain as unilateral VCP rates given the associated symptom variability, inconsistency in performing postoperative laryngeal exam as well as variability in the need to secure the airway surgically. In existing English literature reporting on bilateral VCP, the average rate of permanent bilateral VCP is $45 \%$ (21). The risk of tracheotomy with bilateral VCP averages 30\% with an additional $21 \%$ requiring other acute airway surgery. Thus overall $50 \%$ patients with bilateral VCP require acute airway intervention (21).

Unilateral VCP engenders dysphonia, aspiration, and dysphasia and is associated with ineffective cough and difficulty in lifting maneuvers requiring glottic closure (19-21). Voice changes may be significant enough to result in change in vocation (19-21).

A recent large English data set of over 43,000 patients established that VCP occurring during thyroidectomy is independently predictive of hospital readmission, hospitalization for lower respiratory tract infection and increased risk for gastrostomy and tracheostomy placement (21). In a further analysis, on patients with VCP group ( $\mathrm{n}=67$ ) vsersus control group ( $\mathrm{n}=238$ ), VCP was associated with significant $(\mathrm{P}>0.050)$ increase of hospital charges, hospital stay, 


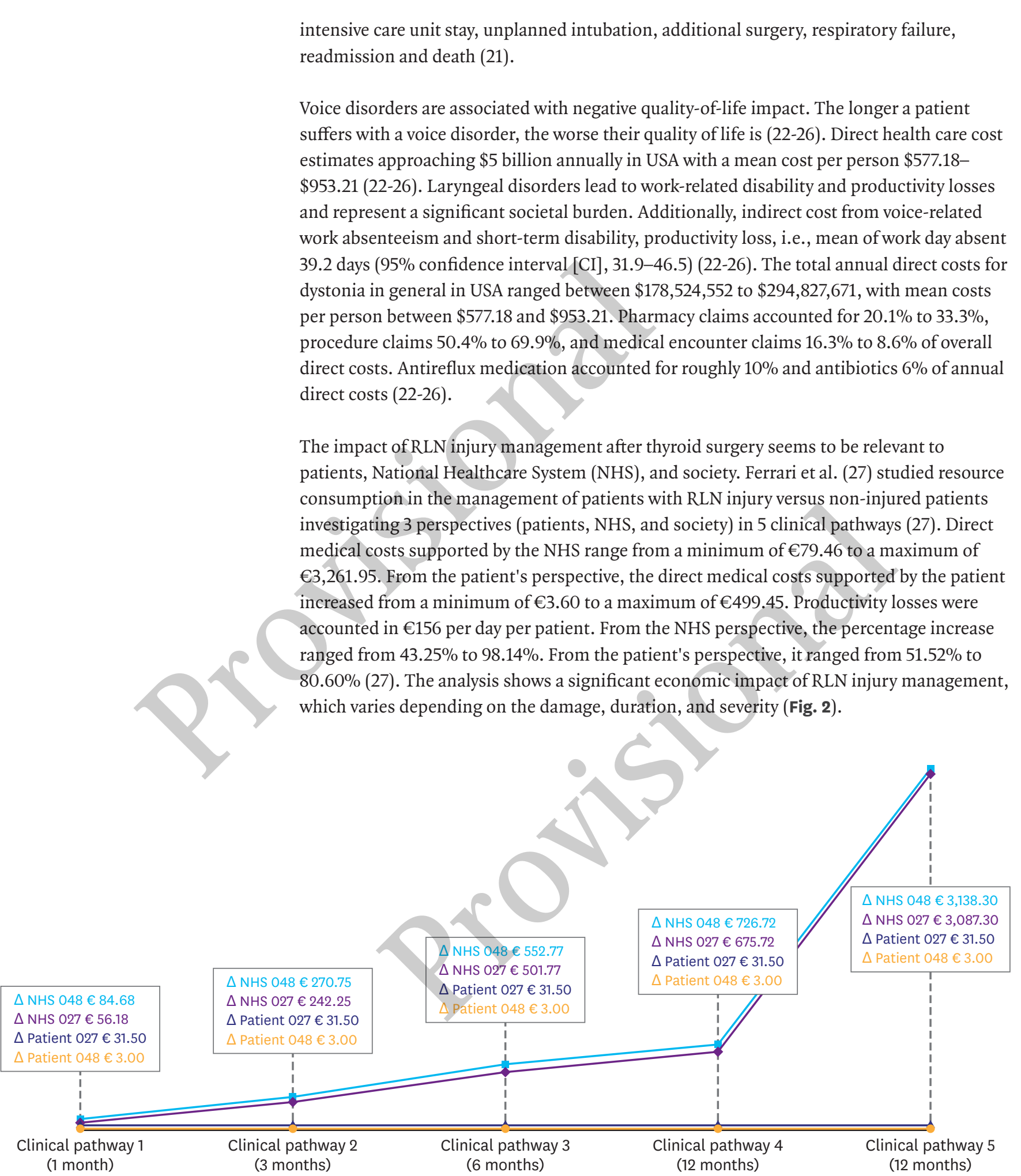

Fig. 2. Cost per RLN injury. Expense are sensitive to time-recovery (i.e., severity) of RLN injury. Figures are higher for thyroid malignant disease than benign surgery. Charges in the case of vocal cord surgery are higher than no medications. Both costs incurred by patient and NHS are significant (figure adapted from reference 27). Each clinical pathway means follow as: clinical pathway 1, RLN function recovery $<1$ month; clinical pathway 2 , RLN function recovery $\geq 1$ month and $<3$ months; clinical pathway 3 , RLN function recovery $\geq 3$ months and < 6 months; clinical pathway 4 , RLN function recovery $\geq 6$ months and < 12 months; clinical pathway 4, RLN function recovery $\geq 12$ months, with phono surgery. $\mathrm{RLN}=$ recurrent laryngeal nerve; $\mathrm{NHS}=$ National Healthcare System. 


\section{PRICE FOR LARYNGEAL EXAMINATIONS}

\section{Timing}

It is interesting to note that in the Literature there are papers that have analyzed the costs of laryngoscopic evaluations.

A study sought to determine how increased time from first primary care to first otolaryngology outpatient visit affected the health care costs of patients with laryngeal/voice disorders. Increased time from first primary care to first otolaryngology evaluation was associated with increased outpatient health care costs. Earlier laryngeal examinations ( $<1$ month) for voice disorders significantly reduce health care expenditures in the evaluation and management of patients with laryngeal/voice disorders. A generalized linear regression model revealed that, compared with patients who saw an otolaryngologist $\leq 1$ month after the first primary care visit, patients in the $>1$ month and $\leq 3$ months and $>3$ month time periods had relative mean cost increases of $\$ 271.34$ (95\% CI, \$115.95-\$426.73) and \$711.38 (95\% CI, \$428.43-\$993.34), respectively (28).

\section{Routine vs. selective use}

Although routine laryngeal examination (RLE) after thyroidectomy may cost more than selective laryngeal examination (SLE), it permits earlier detection and treatment of VCP and so may be cost-saving in the longer term. A study compared the 2-year costeffectiveness between RLE, SLE with RLE performed at 2 weeks (SLE-2w), 1 month (SLE$1 \mathrm{~m}$ ), and 3 months (SLE-3m) after thyroidectomy in the institution's perspective (29). The case definition was a hypothetical 50-year-old woman who underwent an elective total thyroidectomy for a benign multinodular goiter. A decision-analytic model was constructed to compare the estimated cost-effectiveness between RLE, SLE-2w, SLE-1m, and SLE-3m after a 2-year period. Outcome probabilities, utilities, and costs were estimated from the literature. The threshold for cost-effectiveness was set at US \$50,000/quality-adjusted life-year (QALY). Sensitivity and threshold analyses were used to examine model uncertainty. According to this study, RLE was not cost-effective because its incremental cost-effectiveness ratio to SLE-2w, SLE-1m, and SLE-3m were $\$ 302,755$, \$227,883, and $\$ 247,105$, respectively. RLE was only costeffective when the temporary VCP rate increased $>42.7 \%$ or when the cost of RLE equaled zero. Similarly, SLE-2w was only cost-effective to SLE-3m when dysphonia for temporary VCP at 3 months increased $>39.13 \%$, dysphonia for permanent VCP at 3 months increased $>50.29 \%$, or dysphonia without VCP at 3 months increased $>42.69 \%$. However, none of these scenarios appeared clinically likely. In the Institution's perspective, RLE was not costeffective against the other 3 SLE strategies. Regarding to the optimal timing of SLE, SLE-3m appears to be a reasonable and acceptable strategy because of its relative low overall cost (29).

\section{EXPENDITURE MODELS}

One of the major barriers to IONM acceptance is cost-effectiveness. With tighter constraints on health care spending, many recognize the need to identify and restrict clinical interventions that are not cost-effective. As a result, cost-effectiveness analysis is being used increasingly to assess the relative value of surgical interventions. The perception of surgery as expensive and complex might be a barrier to its widespread acceptance in global health efforts.

Most studies focused solely on economic aspects related to use of IONM in thyroid surgery, without consider its clinical benefits (30-33). 
Loch-Wilkinson et al. (34) reported that the IONM technique can not never be cost-effective if measured as a cost real for lesion of the avoided nerve. In this context, comparative analysis of effectiveness plays a crucial role.

Quantification of the economic value of IONM surgery provides a strong argument for the expansion of monitoring's role in the thyroid surgery. Cost-effectiveness analysis is a systematic approach to assessing the relative value of health care interventions (Fig. 3). IONM technique is being used increasingly to frame clinical policy decisions in surgery. Because of this, surgeons need to understand cost-effectiveness analysis and be prepared to examine these studies critically. However, economic value should not be the only argument for resource allocation - other organizational, ethical, and political arguments can also be made for its inclusion. Some intraoperative IONM features and possible consequences of the injuries of the nerve can not be assessed within the total costs (Table 1).

There are a number of cost-effectiveness arguments that can be applied. Currently 4 studies that evaluated cost-effectiveness of IONM.

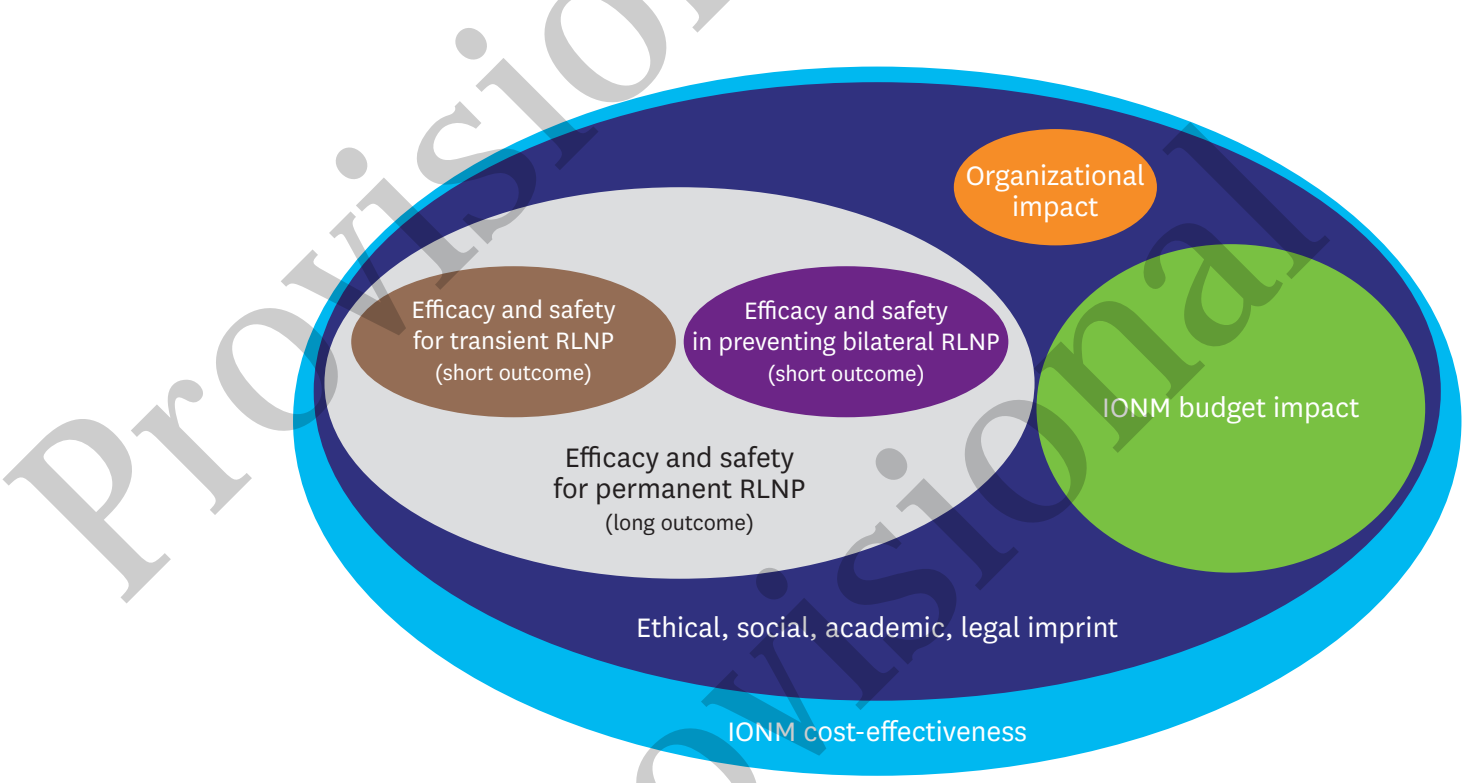

Fig. 3. IONM cost-effectiveness challenges.

IONM = intraoperative neural monitoring; RLNP = recurrent laryngeal nerve palsy.

Table 1. Contents not temperate in IONM cost-effectiveness studies

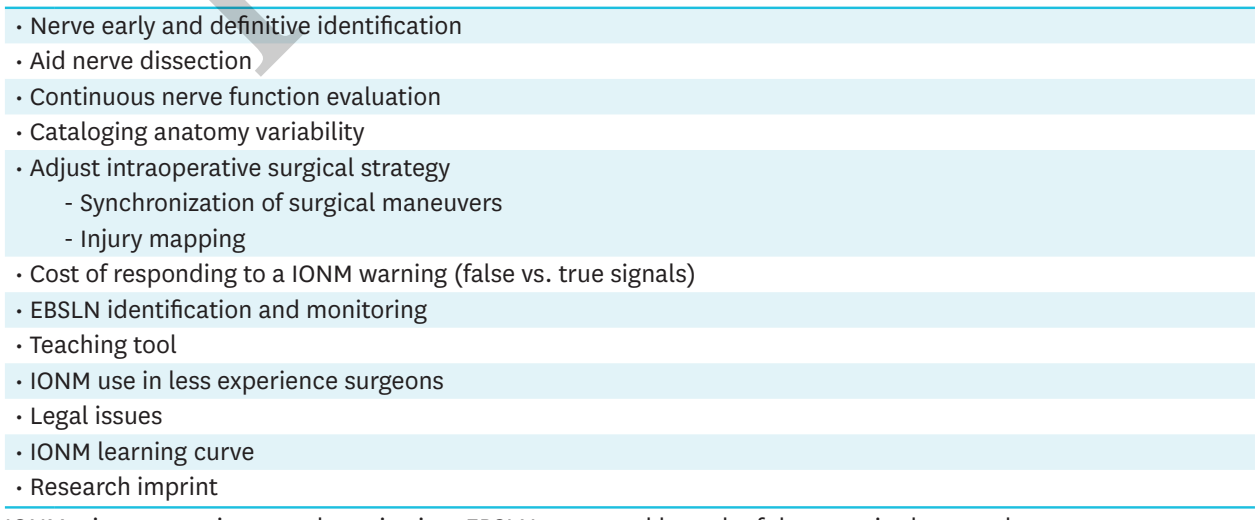

IONM = intraoperative neural monitoring; EBSLN = external branch of the superior laryngeal nerve. 


\section{Paradigm from facial nerve monitoring}

Wilson et al. (35) used a decision analytic cost-effectiveness model and a societal approach to evaluate 3 cohorts of individuals who received 1) intraoperative facial nerve monitoring for both primary and revision middle ear or mastoid surgeries, or 2) facial nerve monitoring for revision surgeries only, or 3) no monitoring for any middle ear or mastoid surgeries. Results strongly favored the use of intraoperative facial nerve monitoring in all patients undergoing middle ear or mastoid surgery, adding about $\$ 222.73$ to $\$ 528.00$ to the total cost. The strategy to monitor primary and revision surgeries had the greatest effectiveness and lowest cost, with an average QALY of 45.68 at an average cost of \$238. Facial nerve monitoring in revision patients only had similar QALYs (45.67) and higher costs (\$292.1). Finally, the strategy not to monitor had the lowest QALY (45.65) and highest cost (\$449.8). The analysis was robust across a wide range of changes in both costs and probabilities.

\section{Stereotype for permeant RLN injuries}

A study used simulation economic modeling to assess clinical and cost-effectiveness utility of IONM implementation in the Italian health care system (30). Wang et al. (30) estimated the cost and consequences of different nerve injury course: 1) no RLN injury, 2) VCP recovery within 1 month, 3) 2 months, 4) 6 months, and 5) after 12 months. In the model applied, the average simulated cohort consisted of a young female patient, 40 years old, employed, daily voice user, who underwent elective, conventional total thyroidectomy via cervical incision using a standardized intermittent IONM technique, for an operable benign, bilateral, diffuse, multinodular, non-toxic, non-retrosternal goiter. IONM was cost-ineffective when parameters such as the rates of transient VCP reached 38.5\%. IONM was cost-effective if the rate of VCP was $33.6 \%$ at 1 month, $22.9 \%$ at 2 months, $9.8 \%$ at 6 months, and $3.8 \%$ at 12 months, independent of phono-surgery. The described scenario is cost-effective only in a high-volume setting. In light of the limitations of a simulation-based study, the Authors conclusively assumed that IONM is cost-effective for permeant RLN injuries (30).

\section{Representation of bilateral thyroid surgery}

In a further analysis, the cost-effectiveness of IONM in preventing bilateral RLN injury was investigated (33). A Markov chain model was constructed based on IONM use. The base-case patient was defined as a 40-year-old woman presenting with a 4.1-cm left-sided papillary thyroid cancer who developed RLN injury with loss of monitoring signal during planned bilateral thyroidectomy. It was hypothesized that, if the surgeon had used IONM, the RLN injury would have been detected and the operation would have been concluded as a thyroid lobectomy to avoid the risk of contralateral RLN injury (33). Cost in US dollars was converted to euros; probabilities and utility scores were identified from the literature and government resources. Length of followup was set as 20 years, and willingness-to-pay (WTP) as $€ 38,000(\$ 50,000)$ per QALY. At the end of year 20, the not using IONM strategy accrued $€ 163,995.40$ (\$215,783.43) and an effectiveness of 14.15 QALYs, whereas use of the IONM strategy accrued $€ 170,283.68(\$ 224,057.48)$ and an effectiveness of 14.33 QALYs. The incremental cost-effectiveness ratio, comparing use versus no use of IONM, was $€ 35,285.26$ ( $\$ 46,427.97)$ per QALY, which is below the proposed WTP, indicating that IONM is the preferred and cost-effective management plan. A Monte Carlo simulation test that considered variability of the main study factors in a hypothetical sample of 10,000 patients showed IONM to be the preferred strategy in $85.8 \%$ of the population (33).

\section{Quotation for IONM selective use in high-risk cases}

Despite the utility of intraoperative nerve monitoring in mapping, early nerve identification, dissection, prognostication and reducing the risk of iatrogenic nerve injury during thyroid 
surgery, its routine use during primary or revision surgery remains controversial. One important question in the current climate of cost-effective medicine is determining which thyroid surgeries should be monitored. One answer to this question is that every case should be monitored because even if there is a chance that a patient could benefit, then some patients will have improved outcomes. In order to identify patients that would benefit the most from approaches traditional surgical procedures or the IONM approach clinical evidence of multicentric studies is needed large-scale or equally rigorous non-randomized evaluations. At the same time, the use of the IONM is likely it will only become routine with subsequent developments in the repayment.

A study creates a decision-tree model of total thyroidectomy to analyze, from a societal perspective, the cost-effectiveness of universal IONM (i.e., use in every case) vs selective IONM (i.e., high-risk cases including reoperative cases, substernal or toxic goiters, and cases with known cancer) vs. no IONM (visual identification only) (32). Parameters for the model were derived from review of the literature, and deterministic and probabilistic analyses were performed to test the model's robustness. All analyses were performed from the model; there were no human participants. Visual identification of the RLN led to a cost savings of $\$ 179.40$ and \$683.20 per patient, and an improvement of 0.001 and 0.004 QALYs, over selective IONM and universal IONM, respectively. Visual identification was the most cost-effective approach, despite variations in costs and utilities in both deterministic and probabilistic sensitivity analyses. In a 1-way sensitivity analysis, decreasing the probability of RLN injury with IONM made selective IONM more cost-effective (32). When the rate of RLN injury for visual identification was kept constant (at 3.86\%), selective IONM became the most costeffective approach when its RLN injury rate dropped below $1.9 \%$. As the rate of RLN injury with IONM dropped below $50.4 \%$ of the visual identification RLN injury rate, selective IONM became the most cost-effective approach. The Authors concluded that visual identification of the RLN is more cost-effective than any use of IONM. If a clinician can, with use of IONM, decrease the rate of RLN injury by $50.4 \%$ or more compared with visual identification, selective use of IONM in high-risk cases is most cost-effective (32).

\section{Juxtaposition}

A study designed a decision analysis to assess the cost-effectiveness of RLN neuromonitoring in the Colombian health system (31). For probabilities, the authors used data from a metaanalysis. Utility was measured using preference values. Authors considered direct costs and conducted a deterministic and a probabilistic analysis. Authors did not find differences in utility between arms. The frequency of RLN injury was $1 \%$ in the neuromonitor group and $1.6 \%$ for the standard group. Thyroidectomy without monitoring was the less expensive alternative. The incremental cost-effectiveness ratio was $\$ 9,112,065$.

\section{ARGUMENTATION}

There has been extraordinary progress in the last 25 years in general surgery. Some events will certainly enter the history of surgery and, among these, conservative surgery in the treatment of breast tumors, minimally invasive laparoscopic surgery or thoracoscopy and the considerable spread of organ transplant surgery. In the field of major oncology surgery, international literature indicates a significant reduction in mortality and morbidity after interventions resection of esophageal, hepatic and pancreatic tumors. 
Certainly the experience, the expertise of the surgical team, the environment in which surgeon operates and the presence in the hospital structure of other specialized skills with which to interact are the prerequisites for obtaining these results, which bear witness to a long and intense basic, translational research, technological, and clinical.

Even the recent history of thyroid surgery is characterized by evolution of progressive acquisitions in different sectors: anatomy, physiology, pathophysiology, genetics, laboratory and radiological diagnostics, and technological innovation with a continuous improvement of the diagnostic and therapeutic approach of thyroid diseases.

In thyroid surgery, in recent years, have been proposed and apply new and interesting technologies. These include surgery minimally invasive, video-assisted or endoscopic, the intraoperative monitoring system of laryngeal nerves, the early perioperative dosage of parathormone for the prevention of symptomatic post-thyroidectomy hypocalcemia, surgical instruments for hemostasis and dissection and finally genetic tests for the detection of patients carrying RET gene mutation.

The new techniques are proposed and immediately applied, without sometimes not allow to measure its effectiveness nor to predict its consequences, positive or negative. It is however considered that their application should be guided by common sense, guaranteed by scrupulous training and, when possible, according to the evidence-based medicine.

\section{REFERENCES}

1. Dralle H, Sekulla C, Lorenz K, Brauckhoff M, Machens AGerman IONM Study Group. Intraoperative monitoring of the recurrent laryngeal nerve in thyroid surgery. World J Surg 2008;32:1358-66. PUBMED | CROSSREF

2. Barczyński M, Konturek A, Cichoń S. Randomized clinical trial of visualization versus neuromonitoring of recurrent laryngeal nerves during thyroidectomy. Br J Surg 2009;96:240-6. PUBMED | CROSSREF

3. Horne SK, Gal TJ, Brennan JA. Prevalence and patterns of intraoperative nerve monitoring for thyroidectomy. Otolaryngol Head Neck Surg 2007;136:952-6. PUBMED | CROSSREF

4. Sturgeon C, Sturgeon T, Angelos P. Neuromonitoring in thyroid surgery: attitudes, usage patterns, and predictors of use among endocrine surgeons. World J Surg 2009;33:417-25. PUBMED | CROSSREF

5. Lamade W, Fogel W, Rieke K, Senninger N, Herfarth C. Intraoperative monitoring of the recurrent laryngeal nerve. A new method. Chirurg 1996;67:451-4. PUBMED

6. Thomusch O, Sekulla C, Walls G, Machens A, Dralle H. Intraoperative neuromonitoring of surgery for benign goiter. Am J Surg 2002;183:673-8. PUBMED | CROSSREF

7. Chiang FY, Lee KW, Chen HC, Chen HY, Lu IC, Kuo WR, et al. Standardization of intraoperative neuromonitoring of recurrent laryngeal nerve in thyroid operation. World J Surg 2010;34:223-9. PUBMED | CROSSREF

8. Randolph GW, Dralle H, Abdullah H, Barczynski M, Bellantone R, Brauckhoff M, et al. Electrophysiologic recurrent laryngeal nerve monitoring during thyroid and parathyroid surgery: international standards guideline statement. Laryngoscope 2011;121 Suppl 1:S1-16. PUBMED | CROSSREF

9. Loch-Wilkinson TJ, Stalberg PL, Sidhu SB, Sywak MS, Wilkinson JF, Delbridge LW. Nerve stimulation in thyroid surgery: is it really useful? ANZ J Surg 2007;77:377-80. PUBMED | CROSSREF 
10. Frick KD. Microcosting quantity data collection methods. Med Care 2009;47:S76-81. PUBMED | CROSSREF

11. Heerey A, McGowan B, Ryan M, Barry M. Microcosting versus DRGs in the provision of cost estimates for use in pharmacoeconomic evaluation. Expert Rev Pharmacoecon Outcomes Res 2002;2:29-33. PUBMED | CROSSREF

12. Dionigi G, Bacuzzi A, Boni L, Rovera F, Dionigi R. What is the learning curve for intraoperative neuromonitoring in thyroid surgery? Int J Surg 2008;6 Suppl 1:S7-12. PUBMED | CROSSREF

13. Antos J, Elwell D. Using activity-based management to control costs \& achieve organization goals. Caring 1998;17:34-8. PUBMED

14. Regione Lombardia (IT). Delibera di Giunta Regionale n. 8501 [Internet]. Milano: Regione Lombardia; 2008 Nov 26 [cited year month day]. Available from: http://www.regione.lombardia.it.

15. Sosa JA, Bowman HM, Tielsch JM, Powe NR, Gordon TA, Udelsman R. The importance of surgeon experience for clinical and economic outcomes from thyroidectomy. Ann Surg 1998;228:320-30. PUBMED | CROSSREF

16. Dionigi G, Bacuzzi A, Boni L, Rausei S, Rovera F, Dionigi R. Visualization versus neuromonitoring of recurrent laryngeal nerves during thyroidectomy: what about the costs? World J Surg 2012;36:748-54. PUBMED | CROSSREF

17. Sanguinetti A, Parmeggiani D, Lucchini R, Monacelli M, Triola R, Avenia S, et al. Intraoperative recurrent laryngeal nerve monitoring in thyroid surgery evaluation of its use in terms of "spending review". Ann Ital Chir 2014;85:418-21. PUBMED

18. Echeverri A, Flexon PB. Electrophysiologic nerve stimulation for identifying the recurrent laryngeal nerve in thyroid surgery: review of 70 consecutive thyroid surgeries. Am Surg 1998;64:328-33. PUBMED

19. Jeannon JP, Orabi AA, Bruch GA, Abdalsalam HA, Simo R. Diagnosis of recurrent laryngeal nerve palsy after thyroidectomy: a systematic review. Int J Clin Pract 2009;63:624-9.

PUBMED | CROSSREF

20. Francis DO, Randolph G, Davies L. Nationwide variation in rates of thyroidectomy among US medicare beneficiaries. JAMA Otolaryngol Head Neck Surg 2017;143:1122-5. PUBMED | CROSSREF

21. Nouraei SA, Allen J, Kaddour H, Middleton SE, Aylin P, Darzi A, et al. Vocal palsy increases the risk of lower respiratory tract infection in low-risk, low-morbidity patients undergoing thyroidectomy for benign disease: a big data analysis. Clin Otolaryngol 2017;42:1259-66. PUBMED | CROSSREF

22. Cohen SM, Dupont WD, Courey MS. Quality-of-life impact of non-neoplastic voice disorders: a metaanalysis. Ann Otol Rhinol Laryngol 2006;115:128-34.

PUBMED | CROSSREF

23. Cohen SM, Lee HJ, Roy N, Misono S. Pharmacologic management of voice disorders by general medicine providers and otolaryngologists. Laryngoscope, in press 2017. PUBMED | CROSSREF

24. Cohen SM, Kim J, Roy N, Asche C, Courey M. The impact of laryngeal disorders on work-related dysfunction. Laryngoscope 2012;122:1589-94. PUBMED | CROSSREF

25. Cohen SM, Kim J, Roy N, Asche C, Courey M. Direct health care costs of laryngeal diseases and disorders. Laryngoscope 2012;122:1582-8. PUBMED | CROSSREF

26. Cohen SM, Kim J, Roy N, Asche C, Courey M. Prevalence and causes of dysphonia in a large treatmentseeking population. Laryngoscope 2012;122:343-8. PUBMED | CROSSREF

27. Ferrari CC, Rausei S, Amico F, Boni L, Chiang FY, Wu CW, et al. Recurrent laryngeal nerve injury in thyroid surgery: clinical pathways and resources consumption. Head Neck 2016;38:1657-65. PUBMED | CROSSREF

28. Cohen SM, Kim J, Roy N, Courey M. Delayed otolaryngology referral for voice disorders increases health care costs. Am J Med 2015;128:426.e11-8. PUBMED | CROSSREF

29. Lang BH, Wong CK, Tsang RK, Wong KP, Wong BY. Evaluating the cost-effectiveness of laryngeal examination after elective total thyroidectomy. Ann Surg Oncol 2014;21:3548-56. PUBMED | CROSSREF 
30. Wang T, Kim HY, Wu CW, Rausei S, Sun H, Pergolizzi FP, et al. Analyzing cost-effectiveness of neuralmonitoring in recurrent laryngeal nerve recovery course in thyroid surgery. Int J Surg 2017;48:180-8. PUBMED | CROSSREF

31. Sanabria Á, Ramírez A. Economic analysis of routine neuromonitoring of recurrent laryngeal nerve in total thyroidectomy. Biomedica 2015;35:363-71.

PUBMED | CROSSREF

32. Rocke DJ, Goldstein DP, de Almeida JR. A cost-utility analysis of recurrent laryngeal nerve monitoring in the setting of total thyroidectomy. JAMA Otolaryngol Head Neck Surg 2016;142:1199-205. PUBMED | CROSSREF

33. Al-Qurayshi Z, Kandil E, Randolph GW. Cost-effectiveness of intraoperative nerve monitoring in avoidance of bilateral recurrent laryngeal nerve injury in patients undergoing total thyroidectomy. $\mathrm{Br} \mathrm{J}$ Surg 2017;104:1523-31. PUBMED | CROSSREF

34. Sun $\mathrm{H}, \mathrm{Wu} \mathrm{CW}$, Kim HY, Dionigi G. Neural monitoring represent central safety asset for new technologies in thyroid surgery in translational protocols. Am J Surg, in press 2018. PUBMED | CROSSREF

35. Wilson L, Lin E, Lalwani A. Cost-effectiveness of intraoperative facial nerve monitoring in middle ear or mastoid surgery. Laryngoscope 2003;113:1736-45.

PUBMED | CROSSREF 